\title{
Functional Neuroanatomy of Meaning Acquisition from Context
}

\author{
Anna Mestres-Missé ${ }^{1}$, Estela Càmara ${ }^{1,2}$, Antoni Rodriguez-Fornells ${ }^{1,3}$, \\ Michael Rotte $^{2 *}$, and Thomas F. Münte ${ }^{2}$
}

\begin{abstract}
An important issue in language learning is how new words are integrated in the brain representations that sustain language processing. To identify the brain regions involved in meaning acquisition and word learning, we conducted a functional magnetic resonance imaging study. Young participants were required to deduce the meaning of a novel word presented within increasingly constrained sentence contexts that were read silently during the scanning session. Inconsistent contexts were also presented in which no meaning could be
\end{abstract}

assigned to the novel word. Participants showed meaning acquisition in the consistent but not in the inconsistent condition. A distributed brain network was identified comprising the left anterior inferior frontal gyrus (BA 45), the middle temporal gyrus (BA 21), the parahippocampal gyrus, and several subcortical structures (the thalamus and the striatum). Drawing on previous neuroimaging evidence, we tentatively identify the roles of these brain areas in the retrieval, selection, and encoding of the meaning.

\section{INTRODUCTION}

The acquisition of word meaning involves establishing a correspondence between concepts and specific sounds or signs. This mapping is largely arbitrary, that is, the different patterns of sound correspond to the same concept in different languages. It has been estimated that between the ages of 2 and 20 years, on average, 6 to 25 words are learned per day and that high school graduates and college students are in the possession of vocabularies between 40,000 to 100,000 words (Landauer, 1986; Nagy \& Anderson, 1984). Most of these words are learned by exposure to appropriate contexts and without explicit instruction (Nagy, Anderson, \& Herman, 1987; Sternberg, 1987). How this is achieved and what brain processes support meaning acquisition from context is largely unknown. As novel words are constantly created (e.g., slang words, technical words, etc.) and most people are faced with the challenge of learning at least one foreign language, an understanding of word learning at the brain level is of great importance. Behavioral research in language learning, summarized by Bloom (2000), suggests that several cognitive mechanisms are called upon to create word-to-meaning mappings, including memory processes, an appreciation of

\footnotetext{
${ }^{1}$ University of Barcelona, Barcelona, Spain, ${ }^{2}$ Otto von Guericke University, Magdeburg, Germany, ${ }^{3}$ Institució Catalana de Recerca i Estudis Avançats (ICREA), Spain

*Current address: Novartis International AG, CH 4002 Basel, Switzerland.
}

the categories and individuals that make up the external world, syntactic processing, and the ability to understand the beliefs and intentions of the others.

Although word learning in adults and children may be different in several aspects, it still seems useful to briefly consider what is known with respect to word acquisition in children: Children grasp the meaning of new words on the basis of a few incidental exposures, without any explicit training or feedback (Waxman, Philippe, \& Branning, 1999; Rice \& Woodsmall, 1988; Heibeck \& Markman, 1987; Dollaghan, 1985), a process known as "fast mapping." Children, as well as adults, possess a whole object bias, that is, a preference to interpret a novel word as referring to a real-world object (Bloom, 2000; Gillette, Gleitman, Gleitman, \& Lederer, 1999; Markman, 1989; Macnamara, 1972). The first words can be learned through the observation of the world and attention to the intentional acts of word users. This strategy works for concrete nouns and action verbs. For closed class words (e.g., determiners or conjunctions) and abstract nouns, for example, this strategy does not work and has to be supplemented by other language processes as, for instance, syntactic processing (Gillette et al., 1999). Although several studies have focused on the influence of syntactic cues in word learning, little is known about learning from semantic context. The present project is devoted to this issue by examining word-to-meaning mappings constructed via contextual learning.

People do not typically use words in isolation but in the context of sentences. Thus, although associative word 
learning might occur in special circumstances (e.g., rote memorizing of the vocabulary of a second language in school) and has been targeted by neuroscientific investigations (Breitenstein et al., 2005; Breitenstein \& Knecht, 2002), the present study rather tries to study word learning in a more natural fashion. To simulate natural word learning, we use a variant of the so-called human simulation paradigm (Gillette et al., 1999). In such studies, participants are exposed to different sources of information (linguistic and extralinguistic), which have to be used to determine the meaning of a novel word. In a previous study (Mestres-Missé, Rodriguez-Fornells, \& Münte, 2007), we have adapted such a paradigm and recorded event-related brain potentials. A word-learning task (see Table 1) was created that allowed adult participants to derive the meaning of new words presented within three successive sentences. After three exposures, brain potentials to novel words in meaningful contexts were indistinguishable from real words. This acquisition effect was not observed for novel words, for which sentence contexts allowed no meaning derivation. Furthermore, when the learned novel words were presented in isolation, an activation of their corresponding meaning was observed, although this process was slower than for real words. Although this earlier study demonstrated the feasibility of

Table 1. Word-learning Task

$M+$ Condition

Mario always forgets where he leaves the lankey

It was expensive the repair of the lankey

I punctured again the wheel of the lankey

$M-$ Condition

I have bought the tickets for the garty

On the construction-site you must wear a garty

Everyday I buy two loaves of fresh garty

\section{$R$ Condition}

She likes people with nice and clean teeth

In a fight Mary had broken two teeth

After a meal you should brush your teeth

Participants are required to discover the meaning of a novel word at the end of each of three successively presented sentences (each 8 words in length). In the $\mathrm{M}+$ condition, the meaning of the novel word was readily apparent, whereas in the $\mathrm{M}-$ condition, no meaning could be mapped to the novel word, as the three sentences each required a different terminal word. To control for the repetition effects across sentences, real words were used at the end of the sentences in the $\mathrm{R}$ condition. Upon the completion of the three sentences (wordby-word presentation, word duration $=200 \mathrm{msec}$; SOA $=500 \mathrm{msec}$ ), a prompt was shown requiring the participants to think about the "hidden" word in the $\mathrm{M}+$ and $\mathrm{M}-$ conditions or to think of a synonym or a semantic related word in the $\mathrm{R}$ condition. Guessing was encouraged. (Note: English translation of Spanish materials, keeping the Spanish word order) the paradigm for neuroscientific investigations, it did not speak to the functional neuroanatomy of meaning acquisition. Therefore, the present investigation tries to identify the brain regions that mediate meaning acquisition of novel words during contextual learning by functional magnetic resonance imaging (fMRI).

Previous imaging studies suggest that candidate brain regions likely involved in word learning and meaning construction might include (i) the left inferior prefrontal cortex, which has been associated to control of semantic retrieval (Badre \& Wagner, 2002; Wagner, Pare-Blagoev, Clark, \& Poldrack, 2001); (ii) left lateral temporal regions, in particular, the middle temporal gyrus (BA 21), which have been related to the storage of long-term conceptual knowledge (Martin \& Chao, 2001), to lexical-semantic processes (Indefrey \& Levelt, 2004; Baumgaertner, Weiller, \& Buchel, 2002; Ferstl \& von Cramon, 2001; Keller, Carpenter, \& Just, 2001; Damasio, Grabowski, Tranel, Hichwa, \& Damasio, 1996), and the activation of visual forms and word meanings (Hagoort et al., 1999; Pugh et al., 1996; Howard et al., 1992); and (iii) anterior temporal lobes, which have been associated with amodal semantic representations (for a review, see Patterson, Nestor, \& Rogers, 2007). The activation of other prefrontal regions involved in executive control, performance monitoring, and working memory aspects of the word learning task is also expected.

\section{METHODS \\ Participants}

Twelve native Spanish volunteers were financially compensated for their participation in the study (mean age $=$ 24.5 years; range $=19-32 ; 6$ women; all right-handed). The protocol had been approved by the ethical committee of the University of Magdeburg and written consent was obtained from each participant.

\section{Task and Stimulus Materials}

Participants silently read groups of three sentences, each ending in the same novel word, and had to discover the meaning of this word. In addition to a condition in which all three sentences were consistently licensing one meaning for the terminal novel word (henceforth, $\mathrm{M}+$ condition; e.g., 1. "I have bought the tickets for the tankey." 2. "Tonight we agreed to go to the tankey."/ 3. "I buy popcorn before entering the tankey." Tankey = movie theater), a second "M-" condition was created, in which the novel word was not associated with a consistent meaning across the sentence triplet (e.g., 1. "Mario always forgets where he leaves the golmet."/ 2. "On the construction site you must wear a golmet."/ 3. "After a meal you should brush your golmet." Golmet = ?). In a third control condition (R), sentences closed with a real word. Sentences were systematically rotated between the 
three critical conditions across participants by creating different scenarios.

Fifty sentence triplets were presented in each condition. The scenarios were created such that the mean frequency of the appropriate terminal words was the same for each condition (between 50.08 and 50.43 Freq/ million; Sebastian-Galles, Marti, Cuetos, \& Carreiras, 2000). Sentences uniformly had a length of eight words. Novel words respected the phonotactic rules of Spanish and were created changing one or two letters of an existing word.

For each target word, three sentences differing in their contextual constraint were built, with the most highly constrained sentence always presented last. In this way, the meaning of the novel word could be derived progressively. The "cloze probability" of each sentence, that is, the probability that the target word would be used in a sentence completion task (Taylor, 1953), was determined as follows: In a first pretest, the first seven words of each sentence were presented in isolation to 160 first-year psychology students of the University of Barcelona who were to complete the sentence frame with the first appropriate word that came to mind. Mean cloze probability for the final pool of 450 sentences (150 groups of three sentences) was: first sentence (low constraint), 6.45\% $(S D=10.41)$; second sentence (medium constraint), 29.43\% ( $S D=18.20)$; and third sentence (high constraint), 79.40\% ( $S D=15.08)$. In a second pretest, 15 first-year students were required to read the three sentences forming each triplet sequentially and to report the word, which fitted best after reading the three sentences. In $92.8 \%(S D=9.1)$ of the triplets, the meaning intended by the experimenters was recovered.

For the $\mathrm{M}-$ condition, sentences from different triplets were mixed, resulting in a different combination of Sentences 1, 2, and 3. Thus, no meaning could be assigned to the novel word but the relative position of a sentence within a triplet was preserved. For the $\mathrm{R}$ condition, the sentences were presented with the appropriate real word in the terminal position. Each list of 150 triplets was divided into 5 runs comprising 10 triplets of each condition as well as 10 additional fixation trials of 4 sec.

Each run started with a fixation asterisk lasting for $8 \mathrm{sec}$ to allow time for T1 equilibration effects. Each trial began with a fixation cross of 500 msec duration, then sentences were presented word by word in the center of the screen (word duration $=200 \mathrm{msec}$, stimulus-onset asynchrony $[\mathrm{SOA}]=500 \mathrm{msec}$ ). The last (8th) word or novel word was presented in red and had a duration of $500 \mathrm{msec}$. After each triplet, a prompt was presented for $2 \mathrm{sec}$ requiring the participants to think covertly about the hidden word. Fixation trials and triplets from the $\mathrm{M}+, \mathrm{M}-$, and $\mathrm{R}$ conditions were presented in a pseudorandom order. Stimulus presentation was controlled by Presentation 0.60 Software (Neurobehavioral
Systems) and synchronized with MRI data acquisition with an accuracy of $1 \mathrm{msec}$. Stimuli were projected onto a screen and could be viewed by the subject through a mirror system mounted on to the head coil.

Prior to the scanning session, participants were carefully trained outside the scanner using test trials to ensure that they fully understood the task. Scanning began with a 15-min structural scan followed by the 5 functional runs, each of about 10 min duration. A short rest was given between the runs.

As the fMRI design did not allow testing for the correct meaning assignment immediately after each trial, a short behavioral test was carried out after each run. In this test, the terminal novel words from the previous sentences ( $\mathrm{M}+$ and $\mathrm{M}-$ conditions) were either paired with their correct meaning or with an unrelated word. In the $\mathrm{M}$ - related condition, novel words were paired with the meaning on the last sentence of the triplet. The two words of a pair were presented simultaneously in the center of the screen. Participants indicated if the novel word and the second word of a pair were synonymous or not by pressing one of two buttons (10 trials after each block; random SOA of 1000-2000 msec). To ensure that words from the R condition were processed as well, an additional test ("did you see this word in the previous block?") comprising the presentation of 10 words from the $\mathrm{R}$ condition and 10 novel words in pseudorandom order was conducted requiring an old/new decision for each stimulus.

\section{MRI Scanning Methods}

Imaging was performed with a neuro-optimized GE Medical Systems 1.5-Tesla Signa Neurovascular MR scanner with standard quadrature head coil. An MR-compatible response box was used containing two response keys (middle finger and forefinger of the right hand). Conventional high-resolution structural images (3-D FSPGR sequence, 60 slices sagittal, $2.8 \mathrm{~mm}$ thickness) were followed by functional images sensitive to blood oxygenation level-dependent (BOLD) contrast (echo-planar [EPI] $\mathrm{T}_{2}{ }^{*}$-weighted gradient-echo sequence, TR/TE/flip angle $=$ $2000 \mathrm{msec} / 40 \mathrm{msec} / 80^{\circ}$ ). Each functional run consisted of 310 sequential whole-brain volumes comprising 23 axial slices aligned to the plane intersecting the anterior and posterior commissures, $3.125 \mathrm{~mm}$ in-plane resolution, $5 \mathrm{~mm}$ thickness, $1 \mathrm{~mm}$ gap between slices, positioned to cover all the brain. The four first volumes were discarded due to T1 equilibration effects.

\section{Preprocessing}

A series of preprocessing steps were implemented using statistical parametric mapping (SPM99; Friston, Josephs, Rees, \& Turner, 1998; Friston et al., 1995). First, for each subject, functional volumes were phase-shifted in 
time with reference to the first slice to minimize purely acquisition-dependent signal variations across slices. Head-movement artifacts were corrected based on an affine rigid-body transformation with reference to the first image of the first run. Functional data were then averaged and mean functional image was normalized using the EPI-derived Montreal Neurological Institute (MNI) template (ICBM 152) provided by SPM. After an initial 12-parameter affine transformation, an iterative nonlinear normalization was applied using discrete cosine basis functions by which brain warps are expanded in SPM (Ashburner \& Friston, 1999). Resulting normalization parameters derived for the mean image were applied to the whole functional set. Finally, functional EPI volumes were resampled into $4-\mathrm{mm}^{3}$ voxels and then spatially smoothed with an $8-\mathrm{mm}$ full-width halfmaximum isotropic Gaussian kernel to minimize effects of intersubject anatomical differences.

\section{Data Analysis}

The statistical evaluation was based on a least-square estimation using the general linear model by modeling the different conditions with a regressor waveform convolved with a canonical hemodynamic response function. Confounding effects in global mean were removed by proportional scaling, and signal-correlated motion effects were eliminated by including the estimated movement parameters. Contrast images were calculated for each subject. The individual contrast images were entered into a second-level analysis using a one-sample $t$ test. Unless mentioned otherwise, contrasts were threshold at $p<$ .001 (uncorrected) with a cluster extend of 20 contiguous voxels, and only clusters at a $p<.05$ at the cluster (corrected for multiple comparisons within the region of interest) were considered significant (Worsley \& Friston, 1995). The maxima of suprathreshold regions were localized by rendering them onto normalized T1 structural images of the MNI reference brain (Cocosco, Kollokian, Kwan, \& Evans, 1997). Maxima and all coordinates are reported in MNI coordinates, as used by SPM99 and labeled following the Talairach atlas.

To investigate the possible correlations between individual subject behavioral performance and the activation changes of those areas that have been found to be modulated by meaning acquisition, the contrast images (from the contrast $\mathrm{M}+>\mathrm{M}-$ ) were entered into a random effects correlation analysis in SPM99 that highlighted the voxels showing a significant correlation between the correct meaning extraction (expressed as the percentage of correct responses on the word-pair task) and the intensity of task-related BOLD activity. In order to restrict the correlation analysis to those regions active only by $\mathrm{M}+$ when compared to $\mathrm{M}-$, an explicit mask $(p<.05$, uncorrected $)$ created from the contrast $\mathrm{M}+>$ $\mathrm{M}-$ was applied to the random effects correlation analysis. For this analysis, activation clusters at a significance level of $p<.05$, corrected, for multiple comparisons within the region of interest were interpreted.

Maps of parameter estimates ( $\beta$ values) were computed from the generalized linear model to assess the magnitude of activation during each stage of the task. The mean parameter estimate of each regressor was then calculated at the activation maximum. Statistical analyses with planned comparisons (two-sided, pairedsample $t$ tests) were used to test differences $(p<.05)$ between the parameter estimates from the different conditions.

\section{RESULTS}

\section{Behavioral Performance}

In the "word-pair" task following each functional run, words from the $\mathrm{M}+$ condition yielded $69 \pm 13 \%(S D)$ correct responses for related and $44 \pm 18 \%$ correct rejection for the unrelated pairs $\left(d^{\prime}=0.728\right)$. As expected, the performance in the $\mathrm{M}-$ conditions did not differ between related and unrelated pairs ( $42 \pm 18 \%$ related, $\left.46 \pm 23 \% ; d^{\prime}=0.270\right)$. The percentage of correct responses was larger in the $\mathrm{M}+$ condition $[t(11)=$ $4.2, p<.001]$.

Regarding reaction times, significant differences were found for $\mathrm{M}+$ words between related and unrelated pairs $[t(1,11)=-3.8, p<.003$; related: $1444 \pm 188 \mathrm{msec}$; unrelated: $1728 \pm 285 \mathrm{msec}]$. No significant differences between both conditions were encountered in the $\mathrm{M}-$ condition $(t<1)$. Thus, the present results show a moderate degree of meaning acquisition for the novel words in the $\mathrm{M}+$.

In the "old/new judgment" task, the percentage of hits was 78.8\% (SD = 10.6) and the percentage of false alarms was $9.3 \%\left(S D=8.2, d^{\prime}=2.12\right)$.

\section{fMRI Results}

Figure 1A and Table 2 show the comparisons between each condition and rest. The contrast between $\mathrm{M}+$ and rest led to differences in activation in the left inferior frontal gyrus (IFG, BA 45), left middle temporal gyrus (MTG, BA 21), left putamen, right posterior parahippocampal gyrus (BA 19), and left posterior parahippocampal gyrus (BA 19) among other regions. $\mathrm{M}-$ versus rest showed activations in the left IFG (BA 45), left MTG (BA 21), right posterior parahippocampal gyrus (BA 31), left posterior parahippocampal gyrus (BA 19), and left putamen among other regions. For the $\mathrm{R}$ condition, activity in the left MTG (BA 21), left IFG (BA 45), and left precentral gyrus (BA 4) was seen.

The comparison between $\mathrm{M}+$ versus $\mathrm{M}-$ (see Figure $1 \mathrm{~B}$ and Table 3 ) showed increased activation for $\mathrm{M}+$ in the left anterior parahippocampal gyrus (BA 35), left precuneus (BA 31), and left thalamus (dorsomedial nucleus). The reverse comparison, $\mathrm{M}-$ minus $\mathrm{M}+$, 


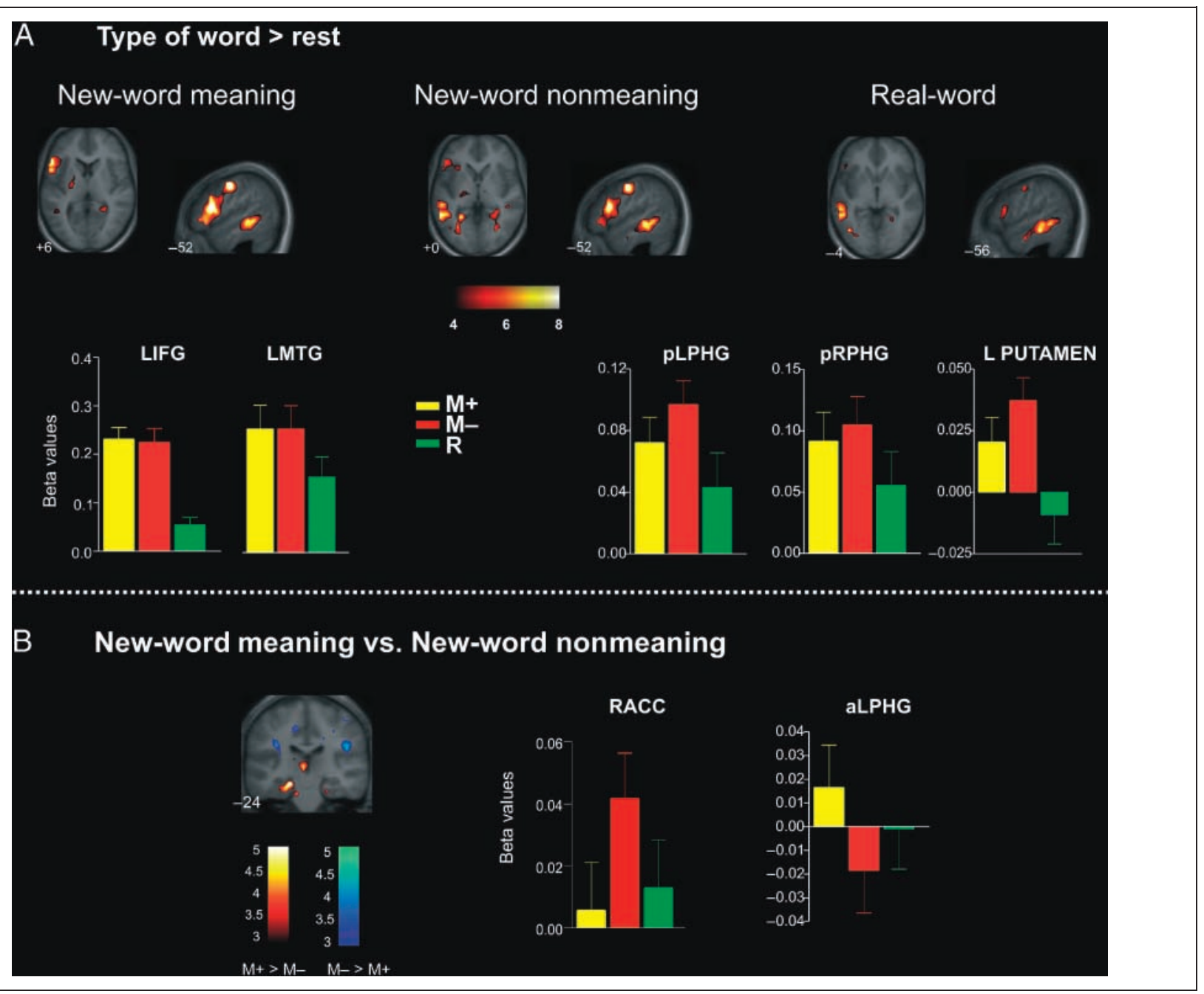

Figure 1. (A) Axial and sagittal views in standard stereotactic space of the group-average comparisons between word type and rest. Notice the differential recruitment of the inferior frontal gyrus (IFG) and the middle temporal gyrus (MTG), as well as hippocampal regions and other subcortical regions, in each condition. All the views presented were superimposed on the mean anatomical image formed averaging for all 12 participants T1 structural MRI scans mapped into normalized MNI space. Values in the color scale refer to the $t$ values of the corresponding contrast. Group-average beta values for $\mathrm{M}+, \mathrm{M}-$, and $\mathrm{R}$ conditions in the left IFG (BA 45, coordinates of the activation maximum: $-56,24$, 12), left MTG (BA 21, coordinates $-60,-36,-8$ ), left posterior parahippocampal gyrus (BA 19, coordinates $-28,-52,0$ ), right posterior parahippocampal gyrus (BA 30, coordinates 28, -48, 4), and left putamen (coordinates -24, -12, 8). Error bars indicate standard error of the mean. (B) Coronal view in standard stereotactic space of the group-average comparisons between $\mathrm{M}+$ and $\mathrm{M}-$ (depicted in red colors) and $\mathrm{M}-$ versus $\mathrm{M}+$ (depicted in blue colors). Notice the larger activation of the left anterior parahippocampal gyrus and the left thalamus (dorsomedial nucleus) in $\mathrm{M}+$, and larger activation in the ACC in $\mathrm{M}-$. View presented was superimposed on the mean anatomical image formed averaging for all 12 participants T1 structural MRI scans mapped into normalized MNI space. Values in the color scale refer to the $t$ values of the corresponding contrast. Group-average beta values for $\mathrm{M}+, \mathrm{M}-$, and $\mathrm{R}$ conditions in the left anterior parahippocampal gyrus (BA 35, coordinates $-20,-24,-16$ ) and the right anterior cingulate gyrus/pre-SMA (BA 32/6, coordinates 12, 8, 40). Error bars indicate standard error of the mean.

showed increased activation in the right insula (BA 13) and right anterior cingulate gyrus (ACC/SMA) (BA 32/6) (see Figure 1B and Table 3).

For the comparison between $\mathrm{M}+$ and $\mathrm{R}$ (see Figure $2 \mathrm{~A}$ ), significant differences in activation were observed in the left IFG (BA 45), the right caudate head, bilateral caudate body, bilateral IFG (BA 47), left thalamus (dorsomedial nucleus), and left precentral gyrus (BA 6) (see Figure 2A and Table 4). The reverse comparison showed activation in the right posterior cingulate gyrus (BA 31), right inferior parietal lobe (BA 40), and left middle frontal gyrus (BA 10) due to the deactivation of these regions in the $\mathrm{M}+$ condition (see Figure $2 \mathrm{C}$ and Table 4 ). When comparing $\mathrm{M}-$ to $\mathrm{R}$, the left IFG (BA 45), right superior frontal gyrus/ACC (BA 8/32), right posterior parahippocampal gyrus (BA 30), bilateral caudate body, right IFG (BA 47), left MTG (BA 21), left posterior parahippocampal gyrus (BA 19), and right precentral gyrus (BA 4) were found activated (see Figure 2A and Table 4). The reverse contrast showed activations in the right precuneus (BA 31), left middle frontal gyrus (BA 10), right superior frontal gyrus/inferior parietal lobe 
Table 2. Brain Regions Showing Changes in Activity for Each Condition, Comparing Type of Word and Rest

\begin{tabular}{|c|c|c|c|c|c|c|}
\hline \multirow[b]{2}{*}{ Brain Region } & \multirow[b]{2}{*}{$\sim B A$} & \multicolumn{4}{|c|}{ Stereotactic Coordinates } & \multirow[b]{2}{*}{$p$ Corrected } \\
\hline & & $x$ & $y$ & $z$ & Z Score & \\
\hline \multicolumn{7}{|l|}{$M+>$ Rest } \\
\hline L IFG & 45 & -56 & 24 & 12 & 5.38 & .001 \\
\hline L MTG & 21 & -60 & -36 & -12 & 4.73 & .001 \\
\hline R Postcentral gyrus & 2 & 52 & -28 & 56 & 4.54 & .001 \\
\hline L Fusiform gyrus & 19 & -44 & -72 & -20 & 4.11 & .001 \\
\hline L Putamen & & -24 & -12 & 8 & 3.94 & .007 \\
\hline R Cerebellum & & 4 & -56 & -44 & 3.90 & .009 \\
\hline L Lingual gyrus & 19 & -32 & -64 & 0 & 3.85 & .007 \\
\hline R Posterior parahippocampal gyrus & 19 & 28 & -48 & 0 & 3.82 & .001 \\
\hline L SFG & 6 & -8 & -8 & 68 & 3.81 & .006 \\
\hline L Posterior parahippocampal gyrus & 19 & -24 & -56 & -4 & 3.51 & .007 \\
\hline \multicolumn{7}{|l|}{$M->$ Rest } \\
\hline L IFG & 45 & -56 & 20 & 20 & 4.97 & .001 \\
\hline L Fusiform gyrus & 19 & -36 & -76 & -20 & 4.79 & .001 \\
\hline L MTG & 21 & -56 & -36 & -4 & 4.75 & .001 \\
\hline L Putamen & & -24 & -12 & 8 & 4.61 & .001 \\
\hline L Posterior parahippocampal gyrus & 19 & -28 & -52 & 0 & 4.51 & .001 \\
\hline R Precentral gyrus & 4 & 36 & -28 & 68 & 4.29 & .001 \\
\hline $\mathrm{R}$ Insula & & 44 & -16 & 16 & 4.07 & .001 \\
\hline R Posterior parahippocampal gyrus & 30 & 28 & -48 & 4 & 4.00 & .001 \\
\hline L Cerebellum & & -44 & -56 & -24 & 3.81 & .010 \\
\hline \multicolumn{7}{|l|}{$R>$ Rest } \\
\hline L MTG & 21 & -56 & -32 & -12 & 4.57 & .001 \\
\hline L Precentral gyrus & 4 & -52 & -8 & 44 & 4.07 & .001 \\
\hline L IFG & 45 & -56 & 20 & 16 & 3.91 & .003 \\
\hline
\end{tabular}

MNI coordinates and $Z$ scores for the peak location in a particular identified anatomical cluster $(p<.001$, uncorrected; 20 voxels spatial extent) for the statistically significant differences of the corresponding activated regions. Note that only the clusters that were significant on a cluster level of $p<.05$ (corrected for multiple comparisons) are listed. $\mathrm{BA}=$ approximate Brodmann's area; $\mathrm{R}=$ right hemisphere; $\mathrm{L}=$ left hemisphere; IFG $=$ inferior frontal gyrus; $\mathrm{SFG}=$ superior frontal gyrus; $\mathrm{MTG}=$ middle temporal gyrus.

(BA 39), and right inferior parietal lobe (BA 40) (see Figure 2C and Table 4).

\section{Parameter Estimates Analysis}

The parameter estimates (beta values) analysis showed that the level of activity for $\mathrm{M}+$ did not differ significantly from $\mathrm{M}$ - for the left IFG, left MTG, left putamen, right posterior parahippocampal gyrus (see Figure 1A), and bilateral caudate (see Figure 2B). The lowest BOLD activity was observed in all of these regions for the
$\mathrm{R}$ condition. By contrast, the right posterior cingulate gyrus showed a reduction of activity of $\mathrm{M}+$ and $\mathrm{M}-$ relative to $\mathrm{R}$ (see Figure $2 \mathrm{C}$ ).

Of particular interest in the present study are those regions that differentiate $\mathrm{M}+$ and $\mathrm{M}-$. Only four regions showed such a pattern: in the left thalamus (dorsomedial nucleus), the $\mathrm{M}+$ condition showed greater activity than both, $\mathrm{M}-[t(11)=5.4, p<.001]$ and $\mathrm{R}$ condition $[t(11)=4.3, p<.001]$. The $\mathrm{M}-$ condition showed also larger activity than the $\mathrm{R}$ condition $[t(11)=2.3, p<.03]$. Moreover, a significant positive correlation was found 
Table 3. Brain Regions Showing Changes in Activity for New-word Conditions, Comparing Meaning and Nonmeaning Conditions

\begin{tabular}{|c|c|c|c|c|c|c|}
\hline \multirow[b]{2}{*}{ Brain Region } & \multirow[b]{2}{*}{$\sim B A$} & \multicolumn{4}{|c|}{ Stereotactic Coordinates } & \multirow[b]{2}{*}{ p Corrected } \\
\hline & & $x$ & $y$ & $z$ & Z Score & \\
\hline \multicolumn{7}{|l|}{$M+>M-$} \\
\hline L Precuneus & 31 & -4 & -68 & 20 & 3.87 & .015 \\
\hline L Anterior parahippocampal gyrus & 35 & -20 & -24 & -16 & 4.14 & $.049 *$ \\
\hline L Thalamus (dorsomedial) & & -4 & -16 & 4 & 3.80 & $.0001 *$ \\
\hline \multicolumn{7}{|l|}{$M->M+$} \\
\hline $\mathrm{R}$ Insula & 13 & 36 & -8 & 28 & 4.42 & .0001 \\
\hline R ACC/pre-SMA & $32 / 6$ & 12 & 8 & 40 & 3.01 & $.013 *$ \\
\hline
\end{tabular}

MNI coordinates and $Z$ score for the peak location in a particular identified anatomical cluster ( $p<.001$, uncorrected; 20 voxels spatial extent) for the statistically significant differences of the corresponding activated regions. Note that only the clusters that were significant on a cluster level of $p<.05$ (corrected for multiple comparisons) are listed. BA = approximate Brodmann's area; $\mathrm{R}=$ right hemisphere; $\mathrm{L}=$ left hemisphere; ACC/ pre-SMA = anterior cingulate gyrus/presupplementary motor area.

$* p<.01 ; 20$ voxels.

between the percentage of correct responses in the word-pair task for $\mathrm{M}+$ (which was taken as an index of meaning acquisition) and the intensity of BOLD response in the thalamus (coordinates of the peak of the cluster, $-8-28$ 8; Pearson $r=.799 ; p<.002$, twotailed) (see Figure 2D). In the left anterior parahippocampal gyrus, a higher level of activation was found for $\mathrm{M}+$ when compared to $\mathrm{M}-[t(11)=5.97, p<.001]$ (see Figure 1B), whereas the reverse pattern was found in the left posterior parahippocampal gyrus $\{\mathrm{M}-$ responses greater than both $\mathrm{M}+[t(11)=-2.1, p<.05]$ and $\mathrm{R}$ $[t(11)=5.4, p<.001]$; no differences between $\mathrm{M}+$ and R\} (see Figure 1A). The right anterior cingulate gyrus again showed a higher level of activation for $\mathrm{M}-$ than $\mathrm{M}+[t(11)=-3.6, p<.004]$ (see Figure $1 \mathrm{~B})$.

In sum, parameter estimates analysis yielded the following pattern: (i) greater activation was observed in the left thalamus and in the left anterior parahippocampal gyrus for $\mathrm{M}+$ compared to $\mathrm{M}-$, (ii) $\mathrm{M}-$ trials showed greater activation than $\mathrm{M}+$ in the left posterior parahippocampal gyrus and right anterior cingulate gyrus. In addition, (iii) a positive correlation across participants between brain activity in the left thalamus and the efficiency of meaning acquisition for novel words was observed.

\section{DISCUSSION}

Using fMRI, the mapping of novel words to meaning derived from sentential context was studied in young adults. The performance in the word-pair task conducted after each functional run indicated that participants were able to acquire the meaning for those novel words that had been read in a set of coherent sentences ( $\mathrm{M}+$ condition) (Chaffin, Morris, \& Seely, 2001). Although performance on that task was far from perfect, it has to be kept in mind that participants were exposed to multiple novel words over a short period of time and further practice would have likely yielded a stronger word-meaning link (Gaskell \& Dumay, 2003).

The fMRI results suggest that word learning is supported by a multi-element brain network including the left IFG (BA 45), the MTG (BA 21), the parahippocampal gyrus, and several subcortical structures. We will consider the possible role of each of these structures in word learning in the following sections.

\section{New Word/Real Word Sentences Processing}

All conditions in the present study showed activation in the medial-temporal cortex, specifically, the MTG (BA 21) and the IFG (BA 45), when compared against fixation control. However, as attested by the parameter estimates, activation was clearly greater for the two novel word conditions, which in turn did not differ.

Several neuroimaging studies suggest that greater middle temporal activation is related to increased semantic integration demands (Baumgaertner et al., 2002; Ni et al., 2000; Price, 2000; Damasio et al., 1996; Just, Carpenter, Keller, Eddy, \& Thulborn, 1996; Martin, Wiggs, Ungerleider, \& Haxby, 1996; Price, Wise, \& Frackowiak, 1996; Vandenberghe, Price, Wise, Josephs, \& Frackowiak, 1996). The middle temporal region is commonly active in semantic tasks independent of input modality, supporting its role in supramodal semantic processing (Price, 2000; Vandenberghe et al., 1996). Applying a semantic integration account to the current data, the fact that no differential effect was found for $\mathrm{M}+$ and $\mathrm{M}-$ conditions might be taken to reflect attempts to integrate candidate concepts that might be represented by the novel word into the sentence context. If this account 


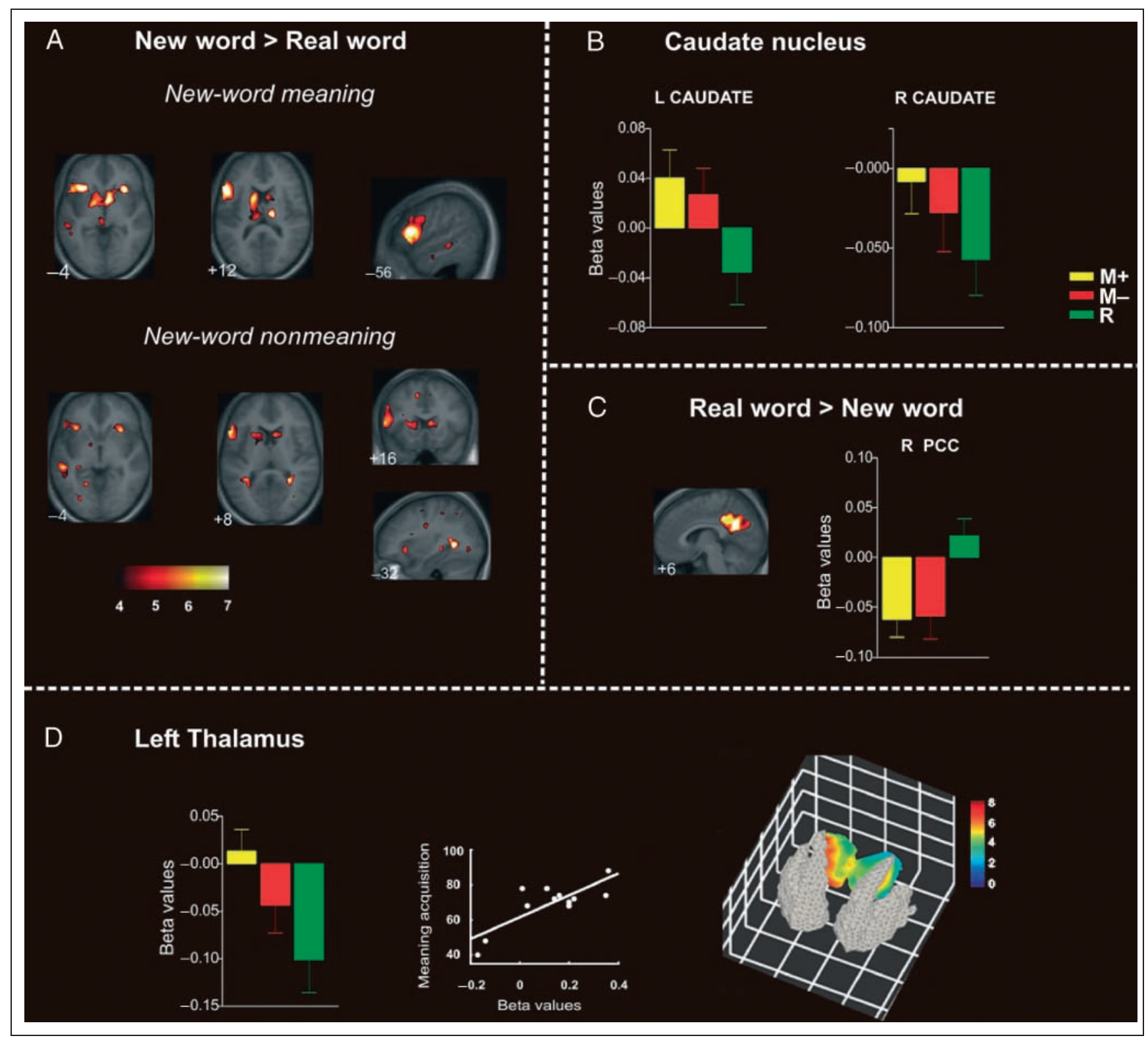

Figure 2. (A) Axial, sagittal, and coronal views in standard stereotactic space of the group-average comparison between new-word conditions versus word. Notice the differential recruitment of subcortical structures in both hemispheres. Sagittal view of the reversed comparison word minus new-word nonmeaning condition. All the views presented were superimposed on the mean anatomical image formed averaging for all 12 participants T1 structural MRI scans mapped into normalized MNI space. Values in the color scale refer to the $t$ values of the corresponding contrast. (B) Group-average beta values for $\mathrm{M}+, \mathrm{M}-$, and $\mathrm{R}$ conditions in the left caudate nucleus (coordinates of the activation maximum: $-12,8,16$ ) and right caudate nucleus (coordinates 8, 20, 0). (C) Group-average beta values for $\mathrm{M}+$, $\mathrm{M}-$, and $\mathrm{R}$ conditions in the right posterior cingulate gyrus (BA 31, coordinates 8, -36, 36). Error bars indicate standard error of the mean. (D) Left: Group-average beta values for $\mathrm{M}+, \mathrm{M}-$ and $\mathrm{R}$ conditions in the left thalamus. Error bars indicate standard error of the mean. Middle: Scatterplot of the beta values against the efficiency of meaning acquisition (corrected recognition rate for $\mathrm{M}+$ ) at the left thalamus (coordinates $-8,-28,8$ ). Right: Tridimensional view of the thalamus based on MARSBAR toolbox (http://marsbar.sourceforge.net/) included templates. Activity maps were constructed by reslicing the original $t$ value map (M+ vs. $\mathrm{M}-)$ to $(1 \times 1 \times 1 \mathrm{~mm})$ and surface-projected to the thalamus-derived template. Radiological convention.

was true, we would predict that, in a longer-term learning study with repeated presentations of $\mathrm{M}+$ words, the activity in this condition should become more similar to the R condition.

Left inferior frontal regions have been implicated in executive aspects during language tasks (Baumgaertner et al., 2002; Wagner et al., 2001; Dapretto \& Bookheimer, 1999; Gabrieli, Poldrack, \& Desmond, 1998; Thompson-
Schill, D'Esposito, Aguirre, \& Farah, 1997; Just et al., 1996). Thus, with an increase in the difficulty of contextual integration, a modulation of the activity in the IFG is predicted. In fact, the anterior region of the left IFG (BA 45) was found more active in both novel word conditions. The IFG is neither morphologically (Amunts et al., 1999) nor functionally homogeneous. The posterior and dorsal part of the left IFG (BA 44) has been impli- 
Table 4. Brain Regions Showing Changes in Activity for Each Condition Comparing New Word and Real Word

\begin{tabular}{|c|c|c|c|c|c|c|}
\hline \multirow[b]{2}{*}{ Brain Region } & \multirow[b]{2}{*}{$\sim B A$} & \multicolumn{4}{|c|}{ Stereotactic Coordinates } & \multirow[b]{2}{*}{ p Corrected } \\
\hline & & $x$ & $y$ & $z$ & Z Score & \\
\hline \multicolumn{7}{|l|}{$M+>R$} \\
\hline L IFG & 45 & -56 & 20 & 12 & 4.75 & $.001 *$ \\
\hline R Caudate head & & 8 & 20 & 0 & 4.32 & $.001 *$ \\
\hline L Caudate body & & -12 & 8 & 12 & 4.21 & $.001 *$ \\
\hline R IFG & 47 & 32 & 24 & -4 & 4.78 & .001 \\
\hline L IFG & 47 & -36 & 24 & -8 & 4.70 & .001 \\
\hline R Caudate body & & 8 & 8 & 12 & 3.84 & .001 \\
\hline L Thalamus (dorsomedial) & & -8 & -20 & 12 & 3.72 & .001 \\
\hline L Precentral gyrus & 6 & -52 & -4 & 52 & 3.55 & .001 \\
\hline \multicolumn{7}{|l|}{$M->R$} \\
\hline L IFG & 45 & -60 & 24 & 12 & 5.06 & .001 \\
\hline R SFG/anterior cingulate & 8 & 4 & 28 & 44 & 4.76 & .001 \\
\hline R Posterior parahippocampal gyrus & 30 & 28 & -48 & 4 & 4.73 & .004 \\
\hline R IFG & 47 & 32 & 20 & -4 & 4.21 & .002 \\
\hline L MTG & 21 & -56 & -32 & -4 & 4.12 & .001 \\
\hline R Caudate body & & 8 & 12 & 12 & 4.08 & .001 \\
\hline L Posterior parahippocampal gyrus & 19 & -20 & -56 & -4 & 3.98 & .001 \\
\hline R Precentral gyrus & 4 & 36 & -20 & 56 & 3.81 & .001 \\
\hline L Caudate body & & -12 & 8 & 16 & 3.66 & .001 \\
\hline \multicolumn{7}{|l|}{$R>M+$} \\
\hline R Cingulate gyrus & 31 & 8 & -36 & 36 & 4.81 & .001 \\
\hline R PLi & 40 & 56 & -36 & 28 & 4.46 & .001 \\
\hline L MFG & 10 & -8 & 48 & 12 & 4.31 & .001 \\
\hline \multicolumn{7}{|l|}{$R>M-$} \\
\hline R Precuneus & 31 & 12 & -60 & 28 & 5.19 & .001 \\
\hline L MFG & 10 & -4 & 56 & 4 & 4.63 & .001 \\
\hline R STG/PLi & 39 & 52 & -56 & 24 & 4.27 & .020 \\
\hline R PLi & 40 & 60 & -36 & 36 & 4.11 & .009 \\
\hline
\end{tabular}

MNI coordinates and $Z$ score for the peak location in a particular identified anatomical cluster $(p<.001$ uncorrected; 20 voxels spatial extent) for the statistically significant differences of the corresponding activated regions. Note that only the clusters that were significant on a cluster level of $p<.05$ (corrected for multiple comparisons) are listed. BA = approximate Brodmann's area; $\mathrm{L}=$ left hemisphere; $\mathrm{R}=$ right hemisphere; IFG $=$ inferior frontal gyrus; $\mathrm{SFG}=$ superior frontal gyrus; MTG = middle temporal gyrus; STG = superior temporal gyrus; PLi = inferior parietal lobule.

${ }^{*} p<.0001 ; 20$ voxels.

cated in interference resolution (Gold et al., 2006; Thompson-Schill et al., 1997), phonological control (Gold \& Buckner, 2002; Fiez, 1997; Fiez, Raichle, Balota, Tallal, \& Petersen, 1996), and syntactic processing (Friederici, Ruschemeyer, Hahne, \& Fiebach, 2003; Friederici, Opitz,
\& von Cramon, 2000; Dapretto \& Bookheimer, 1999), whereas its anterior and ventral regions (BA 47/45) have been associated with semantic control (Gold et al., 2006; Bokde, Tagamets, Friedman, \& Horwitz, 2001; Wagner et al., 2001; Poldrack et al., 1999; Fiez, 1997). 
The results from the present experiment are consistent with previous neuroimaging studies, as the anterior left IFG (pars triangularis, BA 45 and pars orbitalis, BA 47) showed increased activation for those conditions, requiring more elaborate semantic processing (Warburton et al., 1996; Demb et al., 1995; Kapur et al., 1994; Petersen, Fox, Posner, Mintun, \& Raichle, 1988). Although involvement of the IFG in semantic processing was initially unexpected as damage to this region of the brain is not associated with semantic deficits, neuroimaging studies suggest that it is involved in the control of semantic information retrieval (Fiez, 1997; Buckner, Raichle, \& Petersen, 1995; Kapur et al., 1994). Another proposal states that the IFG is required for semantic tasks that require selection from competing semantic features (Thompson-Schill et al., 1997).

The current findings are certainly consistent with these proposals, as in the novel word conditions, a set of initial candidate words that fit the low cloze probability first sentence has to be narrowed down during the second and third sentences. This necessitates controlled retrieval of semantic knowledge and selection of the best fitting candidate concept. With regard to the specific role of the anterior IFG, controlled retrieval versus selection from competing alternatives, one might lean toward the former interpretation, as one should have encountered greater left IFG activation in the $\mathrm{M}-$ condition under the competing alternatives account, because more alternatives compete for selection in this condition.

Bilateral caudate nuclei, as well as the left putamen, showed stronger activation for the novel word conditions (with no differences between $\mathrm{M}+$ and $\mathrm{M}-$ ) than for real word conditions. These structures will be discussed in the next section as they are part of a loop that is, as we will argue, important for meaning acquisition.

Finally, activity decreases occurred in the posterior cingulate and temporo-parietal regions for the $\mathrm{M}+$ and $\mathrm{M}-$ conditions relative to the $\mathrm{R}$ condition. Such differential deactivation has been found previously in language tasks (e.g., Kuperberg et al., 2003). Raichle et al. (2001) have hypothesized that these medial parietal regions are responsible for the continuous gathering of incoming sensory information at rest. Consequently, the differential deactivation of this region, in association with various tasks (including language tasks), should reflect differential attention demands incurred by such tasks. Thus, the higher the attentional demands of a given task, the greater the degree of deactivation in parietal regions. This should also imply that less processing resources are available for other tasks.

\section{Effects of Meaning Acquisition}

A number of subcortical structures showed differential activation depending on the experimental condition, and thus, appear to be involved in word learning. The left thalamus (dorsomedial nucleus) showed greater activation in the $\mathrm{M}+$ condition compared to the $\mathrm{M}-$ and $\mathrm{R}$ conditions; in addition, this region also showed a positive correlation with the efficiency of meaning acquisition. Bilateral caudate nuclei showed enhanced activity in both novel word conditions compared to the real word control sentences. Similarly, the activation of the left putamen was greater in both novel word conditions compared to the $\mathrm{R}$ condition.

The basal ganglia and the thalamus have been shown to play a role in language and cognitive processing. However, despite recent advances in our knowledge about their internal organization and input-output connections (Middleton \& Strick, 2000), the exact computations supported by these structures are not known. Recent fMRI studies have implicated the left thalamus in object recall and lexical retrieval (Crosson et al., 1999, 2003; Kraut et al., 2002). In an experiment by Kraut et al. (2002), participants were required to decide whether two presented object features were related to a specific object (e.g., "desert" and "humps" should activate the concept "camel"). There was thalamic activation when the features elicited object recall in semantic memory, but no activation when the features were merely semantically associated. Further evidence for a role of the left thalamus in language processing comes from patients with isolated anterior thalamic infarcts who have consistently been shown to have word-finding difficulties (Segal, Williams, Kraut, \& Hart, 2003; Ghika-Schmid \& Bogousslavsky, 2000).

With regard to the role of the basal ganglia in language processing, activation of the caudate nucleus or the putamen in language tasks has been inconsistent. Similarly, human lesion data do not provide support for a direct role of either the caudate or the putamen in language (Nadeau \& Crosson, 1997). More recently, Hillis et al. (2004) could demonstrate, in a series of 24 patients with left caudate lesions and aphasia, that the variation of speech and language deficits could be accounted for by variation in the region of cortical hypoperfusion, which was demonstrated by MR perfusion imaging. Aphasia in left striatocapsular strokes thus appears to be due to cortical hypoperfusion caused by large-vessel stenosis that independently caused the left striatocapsular stroke. We will therefore discuss the basal ganglia activations observed in the present study with regard to their possible involvement in the learning aspect of the current task rather than with regard to language processing proper.

These structures have been shown to be part of several parallel loops involving distinct regions of the prefrontal and, to a lesser extent, temporal and parietal cortex (Middleton \& Strick, 2000). Via its participation in these functional loops, the striatum has been related to a number of cognitive and executive processes in addition to the modulation of movements, such as learning, reinforcement, and response selection (Desmond, Gabrieli, \& Glover, 1998). In particular, it seems to play 
an important role in learning arbitrary associations (Laubach, 2005). In a recent study, Crosson et al. (2003) found activation of the left pre-SMA-left dorsal caudateleft ventral anterior thalamic loop in tasks involving retrieval of preexisting lexical items, whether based on semantic or phonological processes.

Several regions of this loop have also been found in the present study. The left thalamus showed greatest activation in the $\mathrm{M}+$ condition (see Figure 2D), whereas the ACC showed greatest activation in the $\mathrm{M}-$ condition (see Figure 1B). The bilateral caudate did not show differences in activation between $\mathrm{M}+$ and $\mathrm{M}-$, but differences when comparing both novel word conditions to real word control sentences (see Figure 2B). These structures appear to facilitate retrieval of lexical items from preexisting stores during language generation and might induce and maintain a processing bias long enough to impact controlled cognitive processes, including working memory (Crosson et al., 2003). We propose that this pattern is consistent with a role of this loop in meaning extraction in the congruent context. Because the thalamus receives inhibition from the globus pallidus, this loop could maintain a bias toward the retrieval of one lexical item versus competing alternatives. Once the most appropriate lexical alternative is selected, inhibition of the thalamus by the globus pallidus might be released via excitation of the caudate nucleus (which increases inhibition of the globus pallidus) and would then lead to thalamic excitation of the frontal cortex (Crosson, Zawacki, Brinson, Lu, \& Sadek, 1997). Consistent with this hypothesis, the ACC shows greater activation in the condition in which meaning could not be extracted (see Figure 1B). The role of the left thalamus in meaning acquisition is further substantiated by a positive correlation between its activity and the efficiency of meaning acquisition (see Figure 2D). To summarize, in the condition in which meaning can be extracted, the bias maintained by the loop discussed above may be overridden, allowing further processing of the selected item by frontal structures.

Moreover, differences in activation between $\mathrm{M}+$ and $\mathrm{M}$ - were found in the medial-temporal lobe. The bilateral posterior parahippocampal gyrus showed enhanced activation of the two novel word conditions compared to the R condition. Prior studies have demonstrated that medial-temporal lobe structures play a central role in associative learning, in episodic memory, and in the acquisition of new lexical information. For example, H. M., a patient characterized with anterograde amnesia following bilateral medial temporal lobe excision, was impaired in the acquisition of new lexical information (Gabrieli, Cohen, \& Corkin, 1988), but not in lexical and grammatical processing tasks (Kensinger, Ullman, \& Corkin, 2001). Importantly, H. M. was not able to learn new words ("xerox") that he had not acquired premorbidly (Gabrieli et al., 1988). Consequently, he also did not show stem completion priming for words that entered the language after the time of his surgery, although normal priming was observed for words that were learned preoperatively (Postle \& Corkin, 1998). This dissociation suggests a role of the medial-temporal lobe in the acquisition of new lexical knowledge (see Ullman, 2001).

Important for the present results, several imaging studies have reported activation of hippocampal and surrounding parahippocampal regions in tasks that required linking the meaning of a target word with context information which provides knowledge about the world (Hoenig \& Scheef, 2005; Bartha et al., 2003; Henke, Weber, Kneifel, Wieser, \& Buck, 1999). Also, a number of recent studies have revealed a role for the parahippocampal gyrus (in particular, on the left) during the comprehension and production of meaningful speech (Awad, Warren, Scott, Turkheimer, \& Wise, 2007) and during listening to and reading of meaningful text (Lindenberg \& Scheef, 2007). Earlier, using depth recordings in epileptic patients undergoing presurgical evaluation, McCarthy, Nobre, Bentin, and Spencer (1995) demonstrated brain potentials that varied as a function of the semantic congruency of the eliciting word to the previous sentence context. This spatial distribution of these negative-field potential suggested that it was generated by the neocortex near the collateral sulcus, including the anterior fusiform and parahippocampal gyri.

In the present study, the posterior parahippocampal gyrus, mostly left, showed greater activation in $\mathrm{M}-$ items than $\mathrm{M}+$ (see Figure 1A). We suggest that the differences observed might reflect semantic associative processing rather than encoding of a retrieved meaning, which should be most pronounced in $\mathrm{M}-$. Because of multiple competing candidates in the $\mathrm{M}-$ condition, the additional activation in this condition can be explained.

Interestingly, the left anterior parahippocampal gyrus displayed greater activation for $\mathrm{M}+$ items than for $\mathrm{M}-$ or $\mathrm{R}$ ones (see Figure 1B). This dissociation between posterior and anterior portions of the parahippocampal cortex is consistent with the proposal that anterior regions within the medial-temporal lobe are predominantly involved in encoding, whereas the posterior regions subserve retrieval (Lepage, Habib, \& Tulving, 1998). This account has received support from several fMRI studies (Prince, Daselaar, \& Cabeza, 2005; Pihlajamaki et al., 2003; Saykin et al., 1999), and the present set of data would certainly be consistent with this proposal as well (anterior: $\mathrm{M}+>\mathrm{M}-\rightarrow$ encoding; posterior $\mathrm{M}->$ $\mathrm{M}+\rightarrow$ retrieval).

\section{Conclusions}

The present study showed a number of brain areas, including subcortical structures, involved in the meaning assignment to novel words. By studying conditions that did or did not allow meaning assignment, we could 
tentatively identify the role of these brain areas in the retrieval, selection, and encoding of the meaning. Obviously, the present study provides only a first glimpse at the neural machinery of word learning. Further manipulations, including, for example, (a) an increased number of exposures to a novel word, (b) a more natural, incidental learning environment with auditory presentation, or (c) the learning of novel words denoting novel concepts, are needed to come to a deeper understanding of the identified network.

\section{Acknowledgments}

This work was supported by grants from the Spanish Government (Ramon y Cajal and MCYT BSO2002-01211) to A. R. F. and a predoctoral grant to A. M. M. and from the DFG to T. F. M. The work was conducted at the Magdeburg Center for Advanced Imaging supported by a BMBF (German Federal Ministry for Education and Research) center grant (contract 01GO0202).

Reprint requests should be sent to Thomas F. Münte, Department of Neuropsychology, Otto von Guericke University, 39106, Magdeburg, Germany, or via e-mail: Thomas.muente@med. ovgu.de.

\section{REFERENCES}

Amunts, K., Schleicher, A., Burgel, U., Mohlberg, H., Uylings, H. B., \& Zilles, K. (1999). Broca's region revisited: Cytoarchitecture and intersubject variability. Journal of Comparative Neurology, 412, 319-341.

Ashburner, J., \& Friston, K. J. (1999). Nonlinear spatial normalization using basis functions. Human Brain Mapping, 7, 254-266.

Awad, M., Warren, J. E., Scott, S. K., Turkheimer, F. E., \& Wise, R. J. (2007). A common system for the comprehension and production of narrative speech. Journal of Neuroscience, 27, 11455-11464.

Badre, D., \& Wagner, A. D. (2002). Semantic retrieval, mnemonic control and prefrontal cortex. Behavioral and Cognitive Neuroscience Reviews, 1, 206-218.

Bartha, L., Brenneis, C., Schocke, M., Trinka, E., Koylu, B., Trieb, T., et al. (2003). Medial temporal lobe activation during semantic language processing: fMRI findings in healthy left- and right-handers. Brain Research, Cognitive Brain Research, 17, 339-346.

Baumgaertner, A., Weiller, C., \& Buchel, C. (2002). Event-related fMRI reveals cortical sites involved in contextual sentence integration. Neuroimage, 16, $736-745$.

Bloom, P. (2000). How children learn the meanings of words. Cambridge: MIT Press.

Bokde, A. L., Tagamets, M. A., Friedman, R. B., \& Horwitz, B. (2001). Functional interactions of the inferior frontal cortex during the processing of words and word-like stimuli. Neuron, 30, 609-617.

Breitenstein, C., Jansen, A., Deppe, M., Foerster, A. F., Sommer, J., Wolbers, T., et al. (2005). Hippocampus activity differentiates good from poor learners of a novel lexicon. Neuroimage, 25, 958-968.

Breitenstein, C., \& Knecht, S. (2002). Development and validation of a language learning model for behavioral and functional-imaging studies. Journal of Neuroscience Methods, 114, 173-179.
Buckner, R. L., Raichle, M. E., \& Petersen, S. E. (1995). Dissociation of human prefrontal cortical areas across different speech production tasks and gender groups. Journal of Neurophysiology, 74, 2163-2173.

Chaffin, R., Morris, R. K., \& Seely, R. E. (2001). Learning new word meanings from context: A study of eye movements. Journal of Experimental Psychology: Learning, Memory, and Cognition, 27, 225-235.

Cocosco, C. A., Kollokian, V., Kwan, R. K. S., \& Evans, A. C. (1997). Online interface to a 3-D MRI simulated brain database. Neuroimage, 5, S425.

Crosson, B., Benefield, H., Cato, M. A., Sadek, J. R., Moore, A. B., Wierenga, C. E., et al. (2003). Left and right basal ganglia and frontal activity during language generation: Contributions to lexical, semantic, and phonological processes. Journal of the International Neuropsychological Society, 9, 1061-1077.

Crosson, B., Sadek, J. R., Bobholz, J. A., Gokcay, D., Mohr, C. M., Leonard, C. M., et al. (1999). Activity in the paracingulate and cingulate sulci during word generation: An fMRI study of functional anatomy. Cerebral Cortex, 9, 307-316.

Crosson, B., Zawacki, T., Brinson, G., Lu, L., \& Sadek, J. R. (1997). Models of subcortical functions in language: Current status. Journal of Neurolinguistics, 10, 277-300.

Damasio, H., Grabowski, T. J., Tranel, D., Hichwa, R. D., \& Damasio, A. R. (1996). A neural basis for lexical retrieval. Nature, 380, 499-505.

Dapretto, M., \& Bookheimer, S. Y. (1999). Form and content: Dissociating syntax and semantics in sentence comprehension. Neuron, 24, 427-432.

Demb, J. B., Desmond, J. E., Wagner, A. D., Vaidya, C. J., Glover, G. H., \& Gabrieli, J. D. (1995). Semantic encoding and retrieval in the left inferior prefrontal cortex: A functional MRI study of task difficulty and process specificity. Journal of Neuroscience, 15, 5870-5878.

Desmond, J. E., Gabrieli, J. D., \& Glover, G. H. (1998). Dissociation of frontal and cerebellar activity in a cognitive task: Evidence for a distinction between selection and search. Neuroimage, 7, 368-376.

Dollaghan, C. (1985). Child meets word: "Fast mapping" in preschool children. Journal of Speech and Hearing Research, 28, 449-454.

Ferstl, E. C., \& von Cramon, D. Y. (2001). The role of coherence and cohesion in text comprehension: An event-related fMRI study. Brain Research, Cognitive Brain Research, 11, 325-340.

Fiez, J. A. (1997). Phonology, semantics, and the role of the left inferior prefrontal cortex. Human Brain Mapping, 5, 79-83.

Fiez, J. A., Raichle, M. E., Balota, D. A., Tallal, P., \& Petersen, S. E. (1996). PET activation of posterior temporal regions during auditory word presentation and verb generation. Cerebral Cortex, 6, 1-10.

Friederici, A. D., Opitz, B., \& von Cramon, D. Y. (2000). Segregating semantic and syntactic aspects of processing in the human brain: An fMRI investigation of different word types. Cerebral Cortex, 10, 698-705.

Friederici, A. D., Ruschemeyer, S. A., Hahne, A., \& Fiebach, C. J. (2003). The role of left inferior frontal and superior temporal cortex in sentence comprehension: Localizing syntactic and semantic processes. Cerebral Cortex, 13, 170-177.

Friston, K. J., Holmes, A. P., Worsley, K. J., Poline, J. B., Frith, C. D., \& Frackowiak, R. S. (1995). Statistical parametric maps in functional imaging: A general linear approach. Human Brain Mapping, 189-210. 
Friston, K. J., Josephs, O., Rees, G., \& Turner, R. (1998). Nonlinear event-related responses in fMRI. Magnetic Resonance in Medicine, 39, 41-52.

Gabrieli, J. D., Cohen, N. J., \& Corkin, S. (1988). The impaired learning of semantic knowledge following bilateral medial temporal-lobe resection. Brain and Cognition, 7, 157-177.

Gabrieli, J. D., Poldrack, R. A., \& Desmond, J. E. (1998). The role of left prefrontal cortex in language and memory. Proceedings of the National Academy of Sciences, U.S.A., 95, 906-913.

Gaskell, M. G., \& Dumay, N. (2003). Lexical competition and the acquisition of novel words. Cognition, 89, 105-132.

Ghika-Schmid, F., \& Bogousslavsky, J. (2000). The acute behavioral syndrome of anterior thalamic infarction: A prospective study of 12 cases. Annals of Neurology, $48,220-227$.

Gillette, J., Gleitman, H., Gleitman, L., \& Lederer, A. (1999). Human simulations of vocabulary learning. Cognition, 73, 135-176.

Gold, B. T., Balota, D. A., Jones, S. J., Powell, D. K., Smith, C. D., \& Andersen, A. H. (2006). Dissociation of automatic and strategic lexical-semantics: Functional magnetic resonance imaging evidence for differing roles of multiple frontotemporal regions. Journal of Neuroscience, 26, 6523-6532.

Gold, B. T., \& Buckner, R. L. (2002). Common prefrontal regions coactivate with dissociable posterior regions during controlled semantic and phonological tasks. Neuron, 35, 803-812.

Hagoort, P., Indefrey, P., Brown, C., Herzog, H., Steinmetz, H., \& Seitz, R. J. (1999). The neural circuitry involved in the reading of German words and pseudowords: A PET study. Journal of Cognitive Neuroscience, 11, 383-398.

Heibeck, T. H., \& Markman, E. M. (1987). Word learning in children: An examination of fast mapping. Child Development, 58, 1021-1034.

Henke, K., Weber, B., Kneifel, S., Wieser, H. G., \& Buck, A. (1999). Human hippocampus associates information in memory. Proceedings of the National Academy of Sciences, U.S.A., 96, 5884-5889.

Hillis, A. E., Barker, P. B., Wityk, R. J., Aldrich, E. M., Restrepo, L., Breese, E. L., et al. (2004). Variability in subcortical aphasia is due to variable sites of cortical hypoperfusion. Brain and Language, 89, 524-530.

Hoenig, K., \& Scheef, L. (2005). Mediotemporal contributions to semantic processing: fMRI evidence from ambiguity processing during semantic context verification. Hippocampus, 15, 597-609.

Howard, D., Patterson, K., Wise, R., Brown, W. D., Friston, K., Weiller, C., et al. (1992). The cortical localization of the lexicons. Positron emission tomography evidence. Brain, 115, 1769-1782.

Indefrey, P., \& Levelt, W. J. (2004). The spatial and temporal signatures of word production components. Cognition, 92, 101-144.

Just, M. A., Carpenter, P. A., Keller, T. A., Eddy, W. F., \& Thulborn, K. R. (1996). Brain activation modulated by sentence comprehension. Science, 274, 114-116.

Kapur, S., Rose, R., Liddle, P. F., Zipursky, R. B., Brown, G. M., Stuss, D., et al. (1994). The role of the left prefrontal cortex in verbal processing: Semantic processing or willed action? NeuroReport, 5, 2193-2196.

Keller, T. A., Carpenter, P. A., \& Just, M. A. (2001). The neural bases of sentence comprehension: A fMRI examination of syntactic and lexical processing. Cerebral Cortex, 11, $223-237$.
Kensinger, E. A., Ullman, M. T., \& Corkin, S. (2001). Bilateral medial temporal lobe damage does not affect lexical or grammatical processing: Evidence from amnesic patient H.M. Hippocampus, 11, 347-360.

Kraut, M. A., Kremen, S., Segal, J. B., Calhoun, V., Moo, L. R., \& Hart, J. (2002). Object activation from features in the semantic system. Journal of Cognitive Neuroscience, 14, $24-36$.

Kuperberg, G. R., Holcomb, P. J., Sitnikova, T., Greve, D., Dale, A. M., \& Caplan, D. (2003). Distinct patterns of neural modulation during the processing of conceptual and syntactic anomalies. Journal of Cognitive Neuroscience, 15, 272-293.

Landauer, T. K. (1986). How much do people remember? Some estimates of the quantity of learned information in long-term memory. Cognitive Science, 10, 477-493.

Laubach, M. (2005). Who's on first? What's on second? The time course of learning in corticostriatal systems. Trends in Neurosciences, 28, 509-511.

Lepage, M., Habib, R., \& Tulving, E. (1998). Hippocampal PET activations of memory encoding and retrieval: The HIPER model. Hippocampus, 8, 313-322.

Lindenberg, R., \& Scheef, L. (2007). Supramodal language comprehension: Role of the left temporal lobe for listening and reading. Neuropsychologia, 45, 2407-2415.

Macnamara, J. (1972). Cognitive basis of language learning in infants. Psychological Review, 79, 1-13.

Markman, E. M. (1989). Categorization and naming in children. Cambridge: MIT Press.

Martin, A., \& Chao, L. L. (2001). Semantic memory and the brain: Structure and processes. Current Opinion in Neurobiology, 11, 194-201.

Martin, A., Wiggs, C. L., Ungerleider, L. G., \& Haxby, J. V. (1996). Neural correlates of category-specific knowledge. Nature, 379, 649-652.

McCarthy, G., Nobre, A. C., Bentin, S., \& Spencer, D. D. (1995). Language-related field potentials in the anterior-medial temporal lobe: I. Intracranial distribution and neural generators. Journal of Neuroscience, 15, 1080-1089.

Mestres-Missé, A., Rodriguez-Fornells, A., \& Münte, T. F. (2007). Watching the brain during meaning acquisition. Cerebral Cortex, 17, 1858-1866.

Middleton, F. A., \& Strick, P. L. (2000). Basal ganglia and cerebellar loops: Motor and cognitive circuits. Brain Research Reviews, 31, 236-250.

Nadeau, S. E., \& Crosson, B. (1997). Subcortical aphasia. Brain and Language, 58, 355-402.

Nagy, W. E., \& Anderson, R. C. (1984). How many words are there in printed English? Reading Research Quarterly, 19, 304-330.

Nagy, W. E., Anderson, R. C., \& Herman, P. A. (1987). Learning word meanings from context during normal reading. American Educational Research Journal, 24, 237-270.

Ni, W., Constable, R. T., Mencl, W. E., Pugh, K. R., Fulbright, R. K., Shaywitz, S. E., et al. (2000). An event-related neuroimaging study distinguishing form and content in sentence processing. Journal of Cognitive Neuroscience, 12, 120-133.

Patterson, K., Nestor, P. J., \& Rogers, T. T. (2007). Where do you know what you know? The representation of semantic knowledge in the human brain. Nature Reviews Neuroscience, 8, 976-987.

Petersen, S. E., Fox, P. T., Posner, M. I., Mintun, M., \& Raichle, M. E. (1988). Positron emission tomographic studies of the cortical anatomy of single-word processing. Nature, 331, 585-589.

Pihlajamaki, M., Tanila, H., Hanninen, T., Kononen, M., Mikkonen, M., Jalkanen, V., et al. (2003). Encoding of 
novel picture pairs activates the perirhinal cortex: An fMRI study. Hippocampus, 13, 67-80.

Poldrack, R. A., Wagner, A. D., Prull, M. W., Desmond, J. E., Glover, G. H., \& Gabrieli, J. D. (1999). Functional specialization for semantic and phonological processing in the left inferior prefrontal cortex. Neuroimage, 10, $15-35$.

Postle, B. R., \& Corkin, S. (1998). Impaired word-stem completion priming but intact perceptual identification priming with novel words: Evidence from the amnesic patient H.M. Neuropsychologia, 36, 421-440.

Price, C. J. (2000). The anatomy of language: Contributions from functional neuroimaging. Journal of Anatomy, 197, 335-359.

Price, C. J., Wise, R. J., \& Frackowiak, R. S. (1996). Demonstrating the implicit processing of visually presented words and pseudowords. Cerebral Cortex, 6, 62-70.

Prince, S. E., Daselaar, S. M., \& Cabeza, R. (2005). Neural correlates of relational memory: Successful encoding and retrieval of semantic and perceptual associations. Journal of Neuroscience, 25, 1203-1210.

Pugh, K. R., Shaywitz, B. A., Shaywitz, S. E., Constable, R. T., Skudlarski, P., Fulbright, R. K., et al. (1996). Cerebral organization of component processes in reading. Brain, 119, 1221-1238.

Raichle, M. E., Macleod, A. M., Snyder, A. Z., Powers, W. J., Gusnard, D. A., \& Shulman, G. L. (2001). A default mode of brain function. Proceedings of the National Academy of Sciences, U.S.A., 98, 676-682.

Rice, M. L., \& Woodsmall, L. (1988). Lessons from television: Children's word learning when viewing. Child Development, 59, 420-429.

Saykin, A. J., Johnson, S. C., Flashman, L. A., McAllister, T. W., Sparling, M., Darcey, T. M., et al. (1999). Functional differentiation of medial temporal and frontal regions involved in processing novel and familiar words: An fMRI study. Brain, 122, 1963-1971.
Sebastian-Galles, N., Marti, N., Cuetos, F., \& Carreiras, M. (2000). LEXESP: Léxico informatizado del español. Barcelona: Edicions de la Universitat de Barcelona.

Segal, J. B., Williams, R., Kraut, M. A., \& Hart, J. (2003). Semantic memory deficit with a left thalamic infarct. Neurology, 61, 252-254.

Sternberg, R. J. (1987). Most vocabulary is learned from context. In M. G. McKeown \& M. E. Curtis (Eds.), The nature of vocabulary acquisition (pp. 89-106). Hillsdale, NJ: Erlbaum.

Taylor, R. L. (1953). Cloze procedure: A new tool for measuring readability. Journalism Quarterly, 30, $415-433$.

Thompson-Schill, S. L., D'Esposito, M., Aguirre, G. K., \& Farah, M. J. (1997). Role of left inferior prefrontal cortex in retrieval of semantic knowledge: A reevaluation. Proceedings of the National Academy of Sciences, U.S.A., 94, 14792-14797.

Ullman, M. T. (2001). The declarative/procedural model of lexicon and grammar. Journal of Psycholinguistic Research, 30, 37-69.

Vandenberghe, R., Price, C., Wise, R., Josephs, O., \& Frackowiak, R. S. (1996). Functional anatomy of a common semantic system for words and pictures. Nature, 383, 254-256.

Wagner, A. D., Pare-Blagoev, E. J., Clark, J., \& Poldrack, R. A. (2001). Recovering meaning: Left prefrontal cortex guides controlled semantic retrieval. Neuron, 31, 329-338.

Warburton, E., Wise, R. J., Price, C. J., Weiller, C., Hadar, U., Ramsay, S., et al. (1996). Noun and verb retrieval by normal subjects. Studies with PET. Brain, 119, 159-179.

Waxman, S. R., Philippe, M., \& Branning, A. (1999). A matter of time: Novel nouns mark object categories when delays are imposed. Developmental Science, 2, 59-66.

Worsley, K. J., \& Friston, K. J. (1995). Analysis of fMRI time-series revisited-Again. Neuroimage, 2, 173-181. 